\title{
The role of quantum effects and non-equilibrium transport coefficients for relativistic heavy ion collisions
}

\author{
M Berenguer†, C Hartnack $\dagger$, G Peilert†, H Stöcker†, W Greiner†, J \\ Aichelin $\ddagger$ and A Rosenhauer $\S$ \\ † Institut für Theoretische Physik, J W Goethe-Universität, D-6000 Frankfurt am Main, \\ Federal Republic of Germany \\ $\ddagger$ Institut für Theoretische Physik, Universität Heidelberg, D-6900 Heidelberg, Federal \\ Republic of Germany \\ $\S$ Department of Physics, University of Bergen, N=5000 Bergen, Norway
}

Received 19 September 1991

\begin{abstract}
Stopping power and thermalization in relativistic heavy ion collisions is investigated employing the quantum molecular dynamics approacl. For heavy systems stopping of the incoming nuclei is predicted, independent of the energy. The influence of the quantum effects and their increasing importance at low energies, is demonstrated by inspection of the mean free path of the nucleons and the $n-n$ collision number. Classical models, which neglect these effects. overestimate the stopping and the thermatization as well as the collective flow and squeeze out. The sensitivity of the transverse and longitudinal momentum transfer to the in-medium cross section and to the pressure is investigated.

The usefulness of thermodynamic concepts, e.g. density, temperature and pressure, is discussed. Local equilibration can be defined only in a fluid picture. II is proven that the projectile and target nuclei do not penetrate into each ofher, as assumed in the two-fluid model. They both collide instead with a 'participan!' component, which consists of those nucleons which have suffered at least one collision. Local equilibration can reach up to about $80 \%$ in each separated fluid.

It is shown that the stress tensor in a one-fluid model cannot be cast in the Newtonian form due to the non-isotropic structure dictated by the initial conditions in relativistic heavy ion collisions. In the 'three-fluid' picture the transverse and longitudinal viscosity coefficien ts have nearly the same magnitude. Thus, both one- and two-fluid viscous hydrodynamic models are not justified microscopically. The three-fluid model and anisotropic hydrodynamics are currently the only macroscopic models which are supported by the microscopic theory.
\end{abstract}

\section{Motivation}

One of the central motivations for studying heavy ion collisions at high energies is the unique opportunity to probe, in the laboratory, hot dense nuclear matter, e.g. the nuclear viscosity and the equation of state. Unfortunately, compression prevails in nuclear collisions only for a very short period and the nucleons continue to interact while the system decompresses. Signatures from the compression stage can be distorted by these final-state interactions. Theoretical studies are needed to find observables linked unambiguously to the densest and most excited state, and are useful for measuring the thermodynamical properties of the system. Furthermore 
the concept of the equation of state is based on local equilibrium. It is therefore an important question as to what degree the hot and compressed nuclear matter thermalizes. This question is most important if one wants to use hydrodynamic concepts to describe the dynamics of heavy ion reactions.

Hydrodynamic calculations revealed that the collective transverse flow probes both the viscosity and the compression energy built up in the collision [1-18]. The transverse flow has been experimentally discovered by the GSI/LBL plastic ball and streamer champer groups [19-29]. It arises as a consequence of the build-up of pressure, which causes the release of the compression energy when the system expands. However, the viscosity strongly damps the collective flow. The high temperature achieved in such reactions, as well as the non-equilibrium and momentum-dependent effects due to the highly anisotropic momentum distribution, diminish the sensitivity of the flow observables to the static part of the nuclear potential.

Now the question arises as to how the system evolves from this highly anisotropic initial stage into the final stage. In order to describe the time evolution of such non-equilibrium processes several distinct microscopic models have been developed. The most common ones are the one-body models of the Vlasov-Uehling-Uhlenbeck (VuU)type [30-39] and the molecular dynamic models, which treat the many-body correlations in a purely classical way [40-49]. This latter type of model has been developed further by the inclusion of the most important quantum effects [50-58] and has been widely used to describe the fragmentation process in nuclear collisions.

In-medium effects like the possible reduction of the nucleon-nucleon scattering cross section [59-61] and the momentum dependence of the nucleon-nucleon interactions (MDI) [62] have an influence on the dynamics. Neither the density dependence of the MDI, nor the correction of the cross section in dense and excited nuclear matter are known and therefore it is certainly necessary to investigate how far the results of theoretical calculations are influenced by both effects. The interplay between the in-medium effects and the equation of state (EOS) on nuclear stopping is also studied in this article.

MDI were first implemented in heavy ion collisions in early studies of molecular dynamics [40, 42, 44] as well as in the multiple scattering model [63] and the timedependent meson field approach [65]. These models predict a significant influence for non-local interactions on the observables: the inclusion of MDI leads to an increase in the collective transverse flow as compared to a local potential alone.

The effective scattering cross section, on the other hand, determines the number of $n-n$ collisions in which the incident kinetic energy is thermalized. The sensitivity of the longitudinal flow to Pauli corrections of the cross sections has recently been demonstrated in vUU $[33,67]$ as well as in QMD $[51,52]$ calculations.

These models have successfully described heavy ion reactions on the one-particle level. However, to compare these with experimental data, the fragmentation process must also be described correctly. It has been shown experimentally [25-29] that multi-fragmentation plays a dominant role in this energy regime. The QMD model can indeed describe the dynamics of multi-fragmentation in this energy regime quite well [51-53]. Recently some attempts have been made to incorporate the fragmentation process in the vuU-type models [67-68].

One of the most important problems in such semiclassical microscopic models is the missing antisymmetrization. The Fermi motion has up until now been incorporated by hand into these models (see, however, [69]). Therefore, the fragments produced have some unrealistic properties. In particular, in the true ground state 
the nucleons would have vanishing momenta. In 1977 Wilets et al [41] had already proposed that this problem could be remedied by simulating the Fermi motion of the nucleons by a quasiclassical momentum-dependent Pauli potential (see also [57, 70]). The inclusion of a Pauli potential strongly influences the EOS at subsaturation densities [71]. It appears necessary to include this effect in the dynamical models $[56-58,71]$.

Non-equilibrium effects can be taken into account in nuclear fluid dynamics (NFD) by means of the viscosity and heat conduction terms, if one assumes that the deviations from local equilibrium are small. Elementary kinetic theory [72-74] connects the viscosity coefficient $\eta$ and the effective scattering cross section $\sigma^{\text {eff }}$ via the relation $\eta \propto 1 / \sigma^{\mathrm{eff}}$, hence the size of $\eta$ directly infuences the amount of nuclear stopping. It has been shown [76] that the nuclear viscosity strongly decreases the transverse flow by a factor of roughly two if $\eta$ is increased from zero (ideal fluid) to $\eta=$ $60 \mathrm{MeV} \mathrm{fm}^{-2} c^{-1}$.

In viscous hydrodynamics a viscosity coefficient of this size is needed in order to match the calculated transverse flow with the data. Values of this order of magnitude have been derived for hot, infinite nuclear matter from the classical kinetic theory [72] and in the Uhlenbeck-Uehling equation [73] and have also been obtained by means of fluid dynamic scaling analysis $[63,64]$.

\section{The QMD model}

The QMD approach, which is described in detail in [51-53], incorporates the important quantum features of the VUU theory [30-39], namely the Pauli principle, stochastic scattering and particle production, into the $N$-body phase space dynamics of the classical molecular dynamics method [40-48].

The nucleons are represented by Gaussians of the form

$$
f_{i}(\boldsymbol{r}, p, t)=\frac{1}{(\pi \hbar)^{3}} \exp \left\{-\frac{\left(\boldsymbol{r}-\boldsymbol{r}_{i 0}(t)\right)^{2}}{2 L}-\left(\boldsymbol{p}-\boldsymbol{p}_{i 0}(t)\right)^{2} \frac{2 L}{\hbar^{2}}\right\}
$$

where $\boldsymbol{r}_{i 0}$ and $\boldsymbol{p}_{i 0}$ are the centroids of particle $i$ in coordinate and momentum space.

The phase space distribution can now be expressed as

$$
f(\boldsymbol{r}, \boldsymbol{p}, t)=\sum_{i=1}^{N} f_{i}(\boldsymbol{r}, \boldsymbol{p}, t)
$$

In terms of these Gaussians the baryon density is given by

$\varrho_{\mathrm{B}}(\boldsymbol{r}, t)=\sum_{i=1}^{N} \int f_{i}(\boldsymbol{r}, \boldsymbol{p}, t) \mathrm{d}^{3} p=\frac{1}{(2 \pi L)^{3 / 2}} \sum_{i=1}^{N} \exp \left[-\left(\boldsymbol{r}-\boldsymbol{r}_{i 0}\right)^{2} / 2 L\right]$.

With the knowledge of the phase space distribution it is possible to calculate thermodynamical quantities locally. The ensemble average of a macroscopic quantity $\chi(r, t)$ is given by

$$
\langle\chi(r, t)\rangle=\frac{1}{\rho(\boldsymbol{r}, l)} \int \mathrm{d}^{3} p \times(p, r, t) f(\boldsymbol{p}, \boldsymbol{r}, t) .
$$


The interactions used here are a local Skyrme two- and three-particle interaction, a Coulomb and a Yukawa interaction. In some cases we also include a MDI which has been adapted from the experimental values of the real part of the proton-nucleus optical potential.

With those Gaussian nucleons, our interactions lead to the following Hamiltonian:

$$
\begin{aligned}
& H=\sum_{i=1}^{N} \frac{p_{i}^{2}}{2 m}+\frac{1}{2} \sum_{i, j=1}^{N}, \int f_{i}\left(\boldsymbol{r}_{i}, p_{i}, t\right) V_{Y u k}^{0} \\
& \times \frac{\exp \left(-\left|\boldsymbol{r}_{i}-\boldsymbol{r}_{j}\right| / \gamma_{Y u k}\right)}{\left|\boldsymbol{r}_{i}-\boldsymbol{r}_{j}\right|} f_{j}\left(\boldsymbol{r}_{j}, \boldsymbol{p}_{j}, t\right) \mathrm{d} \boldsymbol{r}_{i} \mathrm{~d} \boldsymbol{r}_{j} \mathrm{~d} p_{i} \mathrm{~d} \boldsymbol{p}_{j} \\
& +\left(\frac{Z}{A}\right)^{2} \frac{1}{2} \sum_{i, j=1}^{N}, \int f_{i}\left(\boldsymbol{r}_{i}, \boldsymbol{p}_{i}, t\right) \frac{e^{2}}{\left|\boldsymbol{r}_{i}-\boldsymbol{r}_{j}\right|} f_{j}\left(\boldsymbol{r}_{j}, \boldsymbol{p}_{j}, t\right) \mathrm{d} \boldsymbol{r}_{i} \mathrm{~d} \boldsymbol{r}_{j} \mathrm{~d} \boldsymbol{p}_{i} \mathrm{~d} \boldsymbol{p}_{j} \\
& +\frac{1}{2} \sum_{i, j=1}^{N} f f_{i}\left(\boldsymbol{r}_{i}, \boldsymbol{p}_{i}, t\right) \alpha \delta\left(\boldsymbol{r}_{i}-\boldsymbol{r}_{j}\right) f_{j}\left(\boldsymbol{r}_{j}, \boldsymbol{p}_{j}, t\right) \mathrm{d} \boldsymbol{r}_{i} \mathrm{~d} \boldsymbol{r}_{j} \mathrm{~d} \boldsymbol{p}_{i} \mathrm{~d} \boldsymbol{p}_{j} \\
& +\frac{1}{6} \sum_{i, j, k=1}^{N}, \int f_{i}\left(\boldsymbol{r}_{i}, \boldsymbol{p}_{i}, t\right) f_{j}\left(\boldsymbol{r}_{j}, \boldsymbol{p}_{j}, t\right) f_{k}\left(\boldsymbol{r}_{k}, \boldsymbol{p}_{k}, t\right) \\
& \times \beta \delta\left(\boldsymbol{r}_{i}-\boldsymbol{r}_{j}\right) \delta\left(\boldsymbol{r}_{i}-\boldsymbol{r}_{k}\right) \mathrm{d} \boldsymbol{r}_{i} \mathrm{~d} \boldsymbol{r}_{j} \mathrm{~d} \boldsymbol{r}_{k} \mathrm{~d} \boldsymbol{p}_{i} \mathrm{~d} \boldsymbol{p}_{j} \mathrm{~d} \boldsymbol{p}_{k} \\
& +\left(\frac{1}{2} \sum_{i, j=1}^{N}, \int f_{i}\left(r_{i}, p_{i}, t\right) \delta \ln ^{2}\left(\epsilon\left|p_{i}-p_{j}\right|^{2}+1\right)\right. \\
& \left.\times \delta\left(\boldsymbol{r}_{i}-\boldsymbol{r}_{j}\right) f_{j}\left(\boldsymbol{r}_{j}, \boldsymbol{p}_{j}, t\right) \mathrm{d} \boldsymbol{r}_{i} \mathrm{~d} \boldsymbol{r}_{j} \mathrm{~d} \boldsymbol{p}_{i} \mathrm{~d} \boldsymbol{p}_{j}\right) .
\end{aligned}
$$

The evaluation of the integrals yields

$$
\begin{aligned}
& H=\sum_{i=1}^{N} \frac{p_{i}^{2}}{2 m}+\frac{1}{2} \sum_{i, j=1}^{N} \frac{V_{Y u k}^{0}}{2\left|r_{i}-r_{j}\right|} \exp \left(L / \gamma_{Y^{\prime} u k}^{2}\right) \\
& \times\left\{\exp \left(-\left|\boldsymbol{r}_{i}-\boldsymbol{r}_{j}\right| / \gamma_{Y^{\prime} u k}\right)\left[1-\operatorname{erf}\left(\frac{\left(2 L / \gamma_{Y_{u k}}\right)-\left|\boldsymbol{r}_{i}-\boldsymbol{r}_{j}\right|}{\sqrt{4 L}}\right)\right]\right. \\
& \left.-\exp \left(+\left|r_{i}-r_{j}\right| / \gamma_{Y u k}\right)\left[1-\operatorname{erf}\left(\frac{\left(2 L / \gamma_{Y u k}\right)+\left|r_{i}-r_{j}\right|}{\sqrt{4 L}}\right)\right]\right\} \\
& +\left(\frac{Z}{A}\right)^{2} \frac{1}{2} \sum_{i, j=1}^{N}, \frac{e^{2}}{\left|r_{i}-r_{j}\right|} \operatorname{erf}\left(\frac{\left|\boldsymbol{r}_{i}-\boldsymbol{r}_{j}\right|}{\sqrt{4 L}}\right) \\
& +\sum_{i=1}^{N}\left[\frac{\alpha}{2} \frac{1}{(4 \pi L)^{3 / 2} \varrho_{0}} \sum_{j=1}^{N}{ }^{\prime} \exp \left(-\frac{\left(\boldsymbol{r}_{i 0}-\boldsymbol{r}_{j 0}\right)^{2}}{4 L}\right)\right. \\
& \left.+\frac{\beta}{\gamma+1}\left(\frac{1}{(4 \pi L)^{3 / 2} \varrho_{0}} \sum_{j=1}^{N} \exp \left(-\frac{\left(\boldsymbol{r}_{i 0}-\boldsymbol{r}_{j 0}\right)^{2}}{4 L}\right)\right)^{\gamma}\right]
\end{aligned}
$$




$$
\begin{aligned}
& +\sum_{i=1}^{N}\left[\delta \ln ^{2}\left(\epsilon\left|p_{i 0}-p_{j 0}\right|^{2}+1\right) \frac{1}{(4 \pi L)^{3 / 2} \varrho_{0}}\right. \\
& \left.\times \sum_{j=1}^{N} \exp \left(-\frac{\left(\boldsymbol{r}_{i 0}-\boldsymbol{r}_{j 0}\right)^{2}}{4 L}\right)\right] .
\end{aligned}
$$

The primes on the sums indicate that the self-interaction terms are omitted. The three-body part of the Skyrme interaction is here approximated to be proportional to $\varrho^{\gamma}$, in order to allow the compressibility of nuclear matter to be varied as well.

The parameters $\alpha, \beta$ and $\gamma$ are adjusted to reproduce the properties of infinite nuclear matter, i.e.

$$
\begin{aligned}
& \left.\frac{E}{A}\right|_{\varrho=\varrho_{0}}=-16 \mathrm{MeV} \\
& P=\left.\varrho^{2} \frac{\partial E / A}{\partial \varrho}\right|_{\varrho=\varrho_{0}}=0 \mathrm{MeV} \mathrm{^{3 }} \\
& K=\left.9 \varrho^{2} \frac{\partial^{2} E / A}{\partial \varrho^{2}}\right|_{\varrho=\varrho_{0}}= \begin{cases}200 \mathrm{MeV} & \text { (soft EOS) } \\
380 \mathrm{MeV} & \text { (hard EOS). }\end{cases}
\end{aligned}
$$

The parameters of the model are listed in table 1 .

Table 1. Parameters of the modei for the different interactions.

\begin{tabular}{lllllllll}
\hline$K(\mathrm{MeV})$ & EOS & $\alpha(\mathrm{MeV})$ & $\beta(\mathrm{MeV})$ & $\gamma$ & $\delta(\mathrm{MeV})$ & $\epsilon\left(\mathrm{MeV}^{-2}\right)$ & $V_{Y u k}^{0}(\mathrm{MeV})$ & $\gamma_{Y u k}(\mathrm{fm})$ \\
\hline 380 & $\mathrm{H}$ & -124 & 70.5 & 2.0 & - & - & -10 & 1.5 \\
200 & $\mathrm{~S}$ & -356 & 303 & $7 / 6$ & - & - & -10 & 1.5 \\
200 & $\mathrm{SM}$ & -390 & 320 & $8 / 7$ & 1.57 & 21.54 & -10 & 1.5 \\
\hline
\end{tabular}

The short-range interaction is taken into account in the same way as in the cascade and VUU models via a stochastic scattering term: two nucleons can scatter if the spatial distance of the centroids of their Gaussians is smaller than $\sqrt{\sigma_{\text {tot }} / \pi}$. The energy and angular dependence of the experimental differential $n-n$ cross sections $\mathrm{d} \sigma / \mathrm{d} \Omega$ are reproduced. The free $\mathrm{n}-\mathrm{n}$ cross sections are modified in a medium by the Uehling-Uhlenbeck blocking factors $[1-f(r, p)][76]$, which determine the Pauli blocking probability of the final states in an $n-n$ collision. Inclastic processes have also been implemented $[30,55]$.

\section{The importance of the collision term}

Before we investigate the influence of the quantum effects on the reaction dynamics we start with a survey of the time evolution for the reaction $\mathrm{Au}(b=0 \mathrm{fm})+\mathrm{Au}$ 


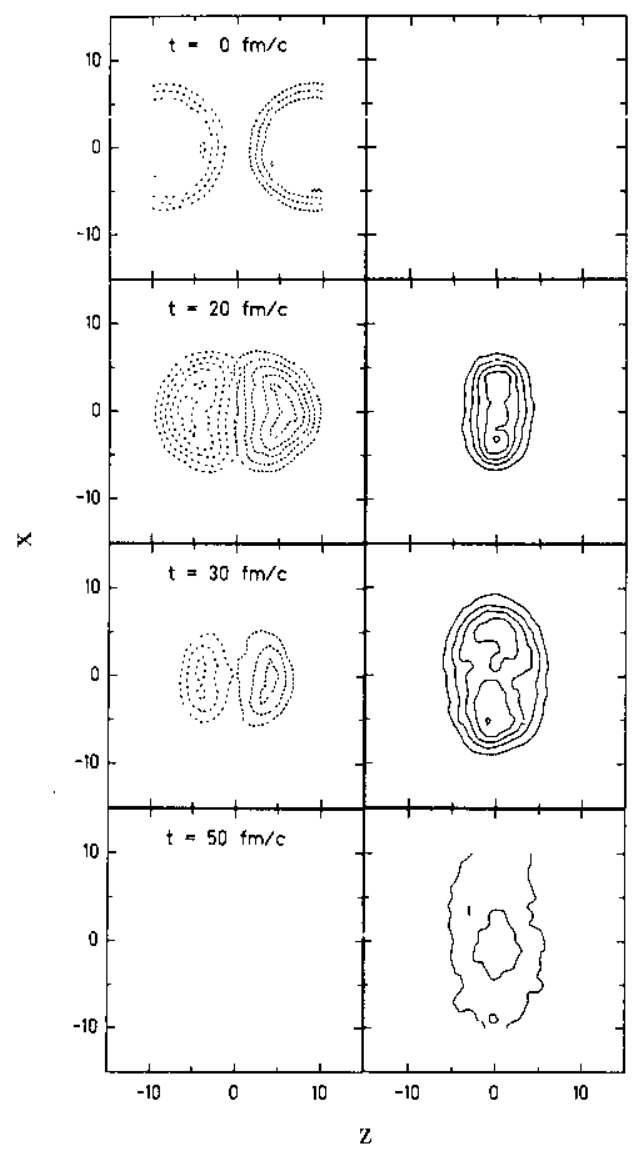

Figure 1. Time evolution of the density contours of the system ${ }^{197} \mathrm{Au}(200 \mathrm{MeV} /$ nucleon, $b=0 \mathrm{fm})+$ ${ }^{197} \mathrm{Au}$ for target and projectile nucleons which have not yet collided (left) and for the 'participant' component (right), which includes all particles which have collided at least once. The contour lines are at $\varrho=0.25,0.5,0.75,1.0,1.25,1.5,1.75,2 \varrho_{0}$. Note that the spectator fluid barely interpenetrates, but instead collides with the participant nucleons piled up in an ellipsoid at midrapidity.

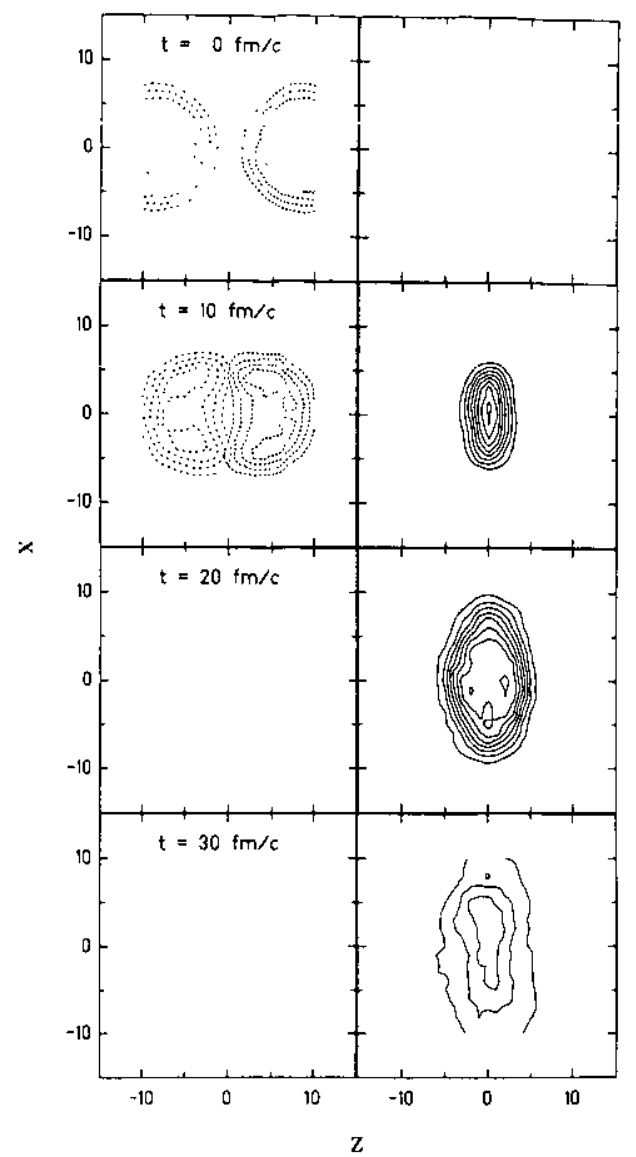

Figure 2. As in figure 1 for the reaction ${ }^{197} \mathrm{Au}$ $(800 \mathrm{MeV} /$ nucleon, $b=0 \mathrm{fm})+{ }^{197} \mathrm{Au}$. At this energy there is no interpenetration of projectile and target.

at 200 and $800 \mathrm{MeV} /$ nucleon bombarding energy. In the following we will define all nucleons which suffered at least one collision as participants.

Figures 1 and 2 show the density profiles of the (cold) projectile and target component in the left-hand column, i.e. all particles that did not collide up to this time. The right-hand column shows the corresponding profiles of the participant component.

It is only at the very beginning of the reaction that there is an overlap between the projectile and target component. The very first collisions (cf figure 8) rapidly build up the participant components. Once these participant components have been 
formed, they act as a buffer between target and projectile-they no longer interact directly. The future evolution of the system is completely determined by the separate interaction of the participant matter with the projectile or target, respectively. Interactions within the participant matter are also crucial. This behaviour is in complete contradiction to the two-fluid model $[4,13,14,16,17]$, where one assumes that the projectile and target component stream through each other and collectively decelerate.

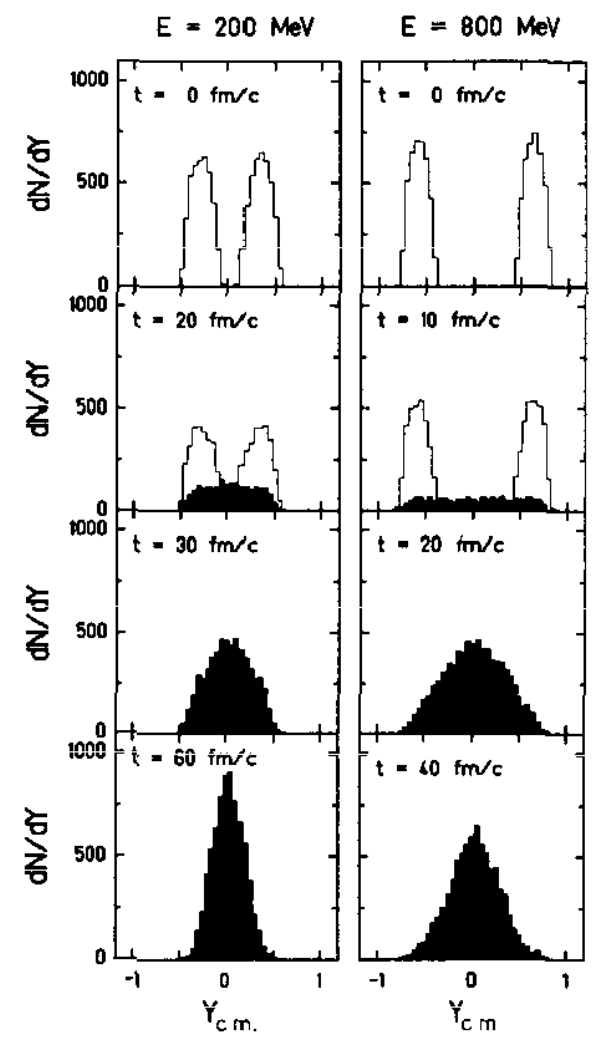

Figure 3. Rapidity distribution $\mathrm{d} N / \mathrm{d} Y^{\prime}$ of the participants (black area) and the spectators (white area) at different reaction times as indicated in central collisions of ${ }^{197} \mathrm{Au}(200$ (left), 800 (right) MeV/nucleon, $b=0 \mathrm{fm}$ ) $+{ }^{197} \mathrm{Au}$. Note that the participants can be distinguished experimentally from the spectators by their rapidities.

The same fact can be observed in momentum space: The $\mathrm{d} N / \mathrm{d} Y$ distribution of the three components is depicted in figure 3. Remnants of the projectile and target do not survive the reaction for $b=0 \mathrm{fm}$. The matter is stopped at the centre of mass rapidity. This supports the three-fluid picture $[9,15]$.

\subsection{The influence of the quantum effects}

The influence of the quantum effects can be studied here by comparing the calculations using the free $n-n$ cross section $\sigma^{\text {free }}$ (without Pauli blocking) to the results including the Uehling-Uhlenbeck scattering cross section [76].

$$
\sigma^{\mathrm{UU}}=\sigma^{\text {frec }}\left(p_{1}, p_{2}, p_{1}^{\prime}, p_{2}^{\prime}\right)\left[1-f\left(r, p_{1}^{\prime}\right)\right]\left[1-f\left(r, p_{2}^{\prime}\right)\right]
$$


$\sigma^{\mathrm{UU}}$ takes into account the Pauli blocking of the final states of two scattered nucleons. The Pauli blocking factors $[1-f(r, p)]$ effectively cause a reduction in the free cross section in the medium. This in turn results in a decrease in the number of collisions.

\subsection{The mean free path}

The influence of this quantum effect on the dynamics of the reaction can most clearly be seen by inspection of the mean free path of the nucleons. The time evolution of all nucleons can be followed in the OMD model. Thus we can 'measure' microscopically the mean free path of all nucleons.

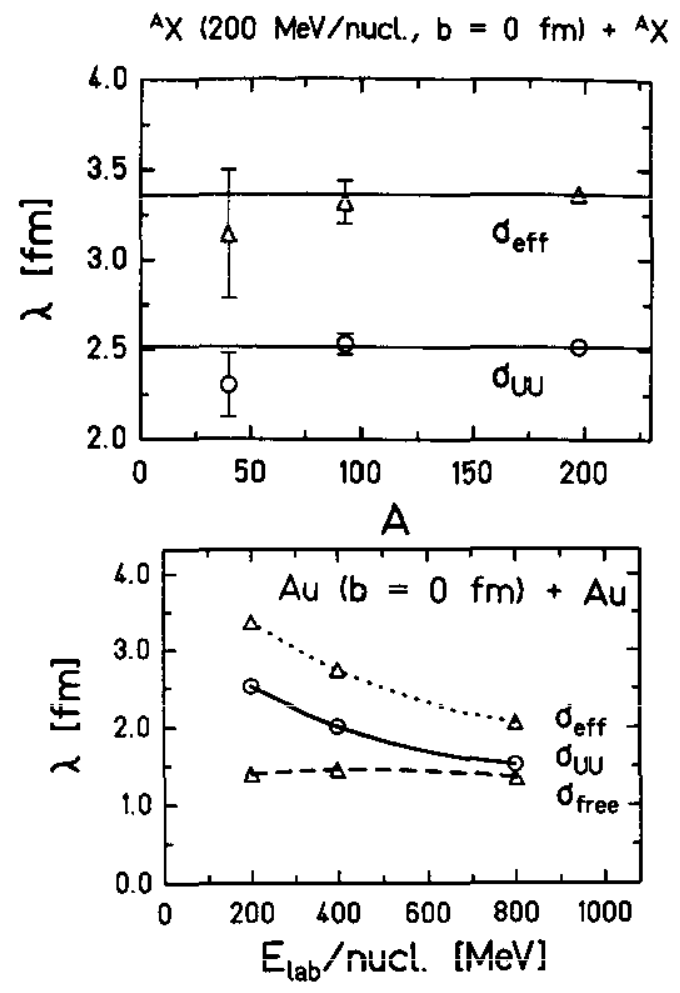

Figure 4. Distribution of the free paths of the nucleons for the reaction ${ }^{197} \mathrm{Au}(200 \mathrm{MeV} /$ nucleon, $b=3 \mathrm{fm})+{ }^{197}$ Au. A large number of nucleons travel only 1-2 $\mathrm{fm} / \mathrm{c}$ between two collisions. This raises doubts as to whether the dilute gas limit is jusîifiêd.

Figure 5. The mean free path of the nucleons is shown for the very central Au + Au collisions at energies from 200 to $800 \mathrm{MeV} /$ nucleon (L) $b=0 \mathrm{fm}$ for the different $n-n$ cross sections, masses and energies as indicated. Note that $\lambda$ does not depend on the masss of the system and decreases with increasing bombarding energy in contrast to the naive expectation of classical kinetic theories [74].

In figure 4 the distribution of the 'free path' $\Delta_{12}$ of the nucleons between subsequent collisions is depicted. One observes a large number of nucleons which travel only 1 or $2 \mathrm{fm} c^{-1}$ between two collisions. There is a small number of nucleons with $\Delta_{12}>10 \mathrm{fm} c^{-1}$. This raises doubts as to whether the dilute gas limit, which is the main assumption of all transport models, and the neglect of three-body collisions is justified. 
Figure 5 (top) shows the dependence of the mean free path on the mass of the system for central, symmetric reactions. Note that the mean free path $\lambda$ is defined as the average of the distribution of figure 4 , taken over all collisions. The results are shown for the Uehling-Uhlenbeck cross section $\sigma^{\mathrm{UU}}$ and for a reduced cross section $\sigma^{\text {eff }}$. Here an overall reduction of $30 \%$, as proposed by Malfliet, Batemans and ter Haar $[59,60]$, has been used in order to take the Pauli blocking of the intermediate scattering states into account. This reduction of the cross section clearly reduces the number of collisions and therefore increases $\lambda$. Note that $\lambda$ depends on the $n-n$ cross section and not on the mass of the system. The energy dependence of $\lambda$ can be studied for the most central collisions of $\mathrm{Au}$ on Au in figure 5 (bottom).

$\lambda$ decreases with increasing bombarding energy, due to the higher densities achieved, in spite of a decreasing $n-n$ cross section. The results obtained with the Boltzmann cross section $\sigma^{\text {free }}$ (without Pauli blocking) is also shown. This increased cross section yields a drastic decrease of the mean free path. Enhanced thermalization and nuclear stopping results.

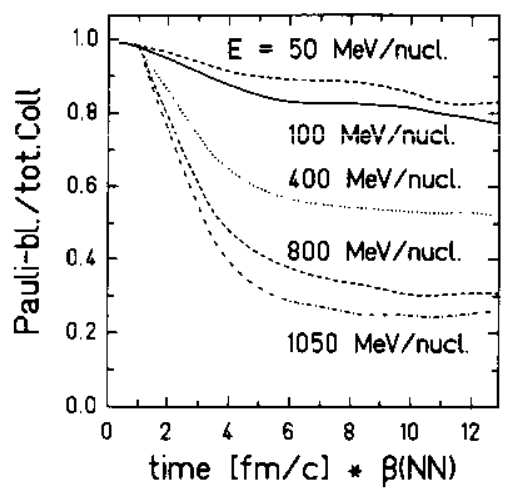

Figure 6. Time dependence of the ratio of Pauli blocked collisions to the total number of attempted collisions at different beam energies for the system ${ }^{93} \mathrm{Nb}(b=3 \mathrm{fm})+{ }^{93} \mathrm{Nb}$, sofi EOS. Observe that Pauli blocking is crucial even for the highest bombarding energies.
Au $(200 \mathrm{MeV} /$ nucl., $b=3 \mathrm{fm})+\mathrm{Au}$
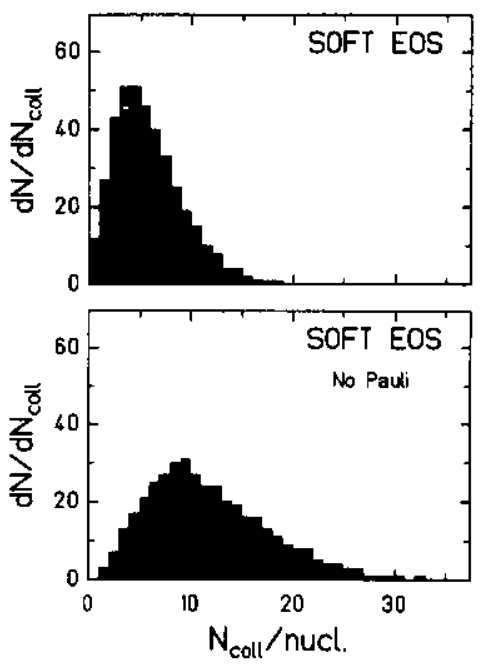

Figure 7. The distribution of the number of collisions is shown for the reaction ${ }^{197} \mathrm{Au}$ (200 MeV/nucleon, $b=3 \mathrm{fm}$ ) $+{ }^{197} \mathrm{Au}$ for the soft EOS with and without Pauli blocking of the final scattering states.

In order to demonstrate the importance of the Pauli blocking, the fraction of the Pauli blocked collisions to all attempted collisions is shown in figure 6 for the system $\mathrm{Nb}+\mathrm{Nb}$ for different beam energies between 50 and $1050 \mathrm{MeV} /$ nucleon The ratio is plotted against $t \beta$. Here $t \beta$ is the scaled reaction time, i.e. $t$ multiplied with the velocity $\beta$ of the incoming projectile in the equal speed system. Ihis product corresponds to the distance travelled by the projectile and target in the $z$-direction. It scales the time according to the velocity of the incoming projectile.

All curves start with a blocking fraction of one. This is due to the fact that all collisions are Pauli blocked in the ground state. After the two nuclei touch each other the blocking factors decrease and saturate after $t \beta \approx 5-6 \mathrm{fm}$. This distance 
corresponds to the total overlap of both nuclei. For very low bombarding energies (the TDHF regime) the blocking factor remains close to one, whereas for higher energies it decreases down to 0.2 at $1 \mathrm{GeV} /$ nucleon.

The distribution of the number of $n-n$ collisions is shown in figure 7 for the reaction $\mathrm{Au}(200 \mathrm{MeV} /$ nucleon, $b=3 \mathrm{fm})+\mathrm{Au}$. This distribution is peaked at about five collisions per nucleon for the soft EOS. The tail, however, shows particles with more than 10-15 collisions. A considerable number of particles have suffered no collisions at all and can therefore be called true spectators. They reside in the nuclear coronas (pole-caps) in these off-centre collisions.

The importance of the quantum effects for the number of collisions is studied in figure 7 (bottom): Without the Pauli blocking the number of collisions doubles and the number of spectators decreases to zero.

Therefore classical molecular dynamic models [40-49], which neglect this quantum effect, overestimate drastically the importance of the $n-n$ collisions, especially at low energies.

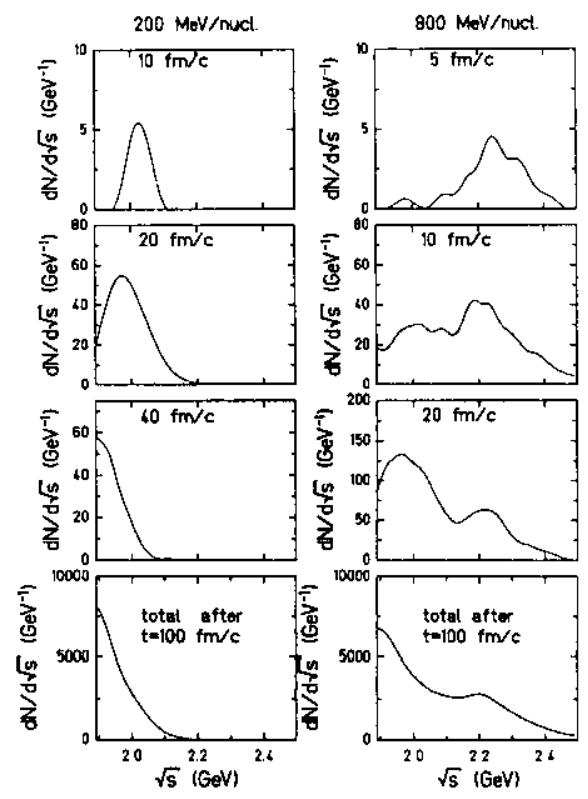

Figure 8. Time dependence of the energy distributions $\mathrm{d} N / \mathrm{d} \sqrt{\mathrm{s}}$ of the $\mathrm{n}-\mathrm{n}$ collisions in the reactions ${ }^{197} \mathrm{Au}$ (200 and $800 \mathrm{MeV} /$ nucleon, $b=3 \mathrm{fm}$ ) $+{ }^{197} \mathrm{Au}$. The beginning of the reaction is clearly governed by hard collisions, while sof collisions dominate further on.

Figure 8 shows the CM energy distributions $\mathrm{d} N / \mathrm{d} \sqrt{s}$ of the $\mathrm{nn}$ collisions, again for the reactions $\mathrm{Au}(200,800 \mathrm{MeV} /$ nucleon, $b=3 \mathrm{fm})+$ Au. The lower row give the total, time integrated, distributions. A peak in the energy distribution is due to the first collisions, which occur roughly at the cnergy $\sqrt{s}=2 m+E_{\mathrm{cm}}^{\mathrm{kin}}$. This underlines the importance of the non-equilibrium effects at the early times.

The time evolution of the scattering process shows that, while the dynamics in the beginning of the reaction is dominated by high energy collisions, the soft collisions dominate subsequently. Therefore, processes which are most sensitive to the hard collisions, such as (subthreshold) particle production $(\pi, \eta, K)$, are mostly influenced by 
the initial projectile and target momentum distribution in the early, non-equilibrium stage of the reaction. The collective flow builds up later, when the system is equilibrated and yields the expansion of the system.

\subsection{The sensitivity of nuclear stopping to the in-medium cross sections}

Let us now inspect in more detail the stopping of the incoming matter which implies the equilibration of the incident longitudinal momenta in $n-n$ collisions.

Au $(200 \mathrm{MeV} / \mathrm{nucl}, \mathrm{b}=3 \mathrm{fm}\}+\mathrm{Au}$

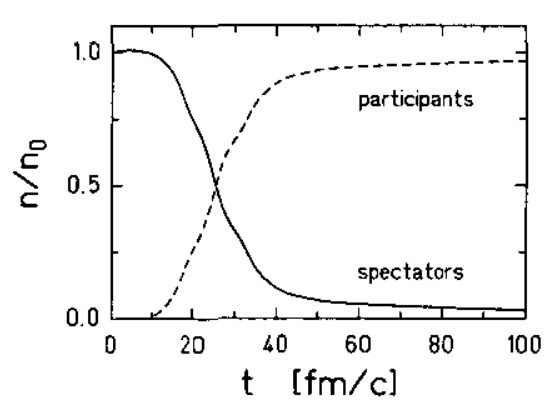

Figure 9. Conversion of the spectators into the participant (fireball) component as a function of time for the same system as in figure 4 . All particles having suffered at least one $n-n$ collision are assumed to belong to the fireball component. $n / n_{0}$ denotes the ratio of nucleons which belong to the spectator (participant) component. Note the rapid onset of the thermalization process as soon as the nuclei touch each other.

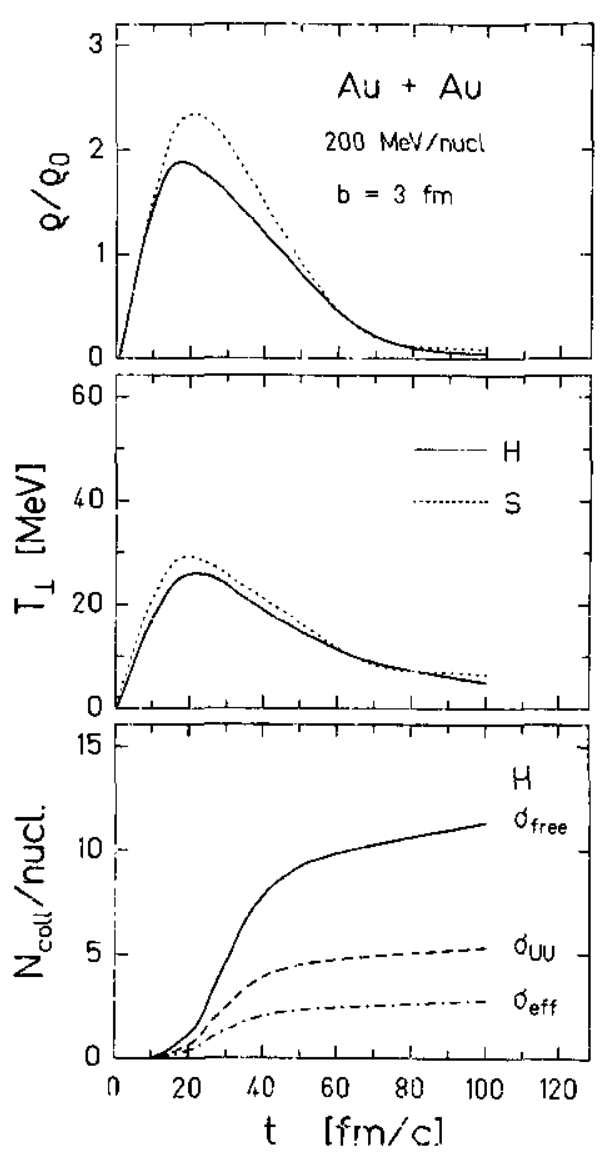

Figure 10. Time evolution of the central compression, the transverse 'temperature' and the average number of collisions per nucleon of the reaction ${ }^{197} \mathrm{Au}$ (200 MeV/nucleon, $\left.b=3 \mathrm{fm}\right)+{ }^{197} \mathrm{Au}$. The local 'temperature' has been obtained via the relation $T=\left\langle p_{\perp}^{2}\right\rangle / 2 m$ lor the different interactions as indicated. Almost all collisions take place when the 'temperature' and densities are at their highest values.

The thermalization process is associated with the conversion of the cold projectile and target matter into the hot participant (fireball) component. The time evolution of this process is depicted in figure 9 for a central collision. Observe the rapid onset of 
the thermalization process, as soon as the nuclei overlap in configuration space. Late in the compression stage almost all nucleons are found in the participant component. This is due to the large number of $n-n$ collisions in the system.

Some quantities of interest for macroscopic models are shown in figure 10 for the reaction $\mathrm{Au}(200 \mathrm{MeV} /$ nucleon, $b=3 \mathrm{fm})+\mathrm{Au}$ as a function of time. The central density increases from zero (in the initial stage the nuclei are separated in coordinate space) to the maximum density reached after $15 \mathrm{fm} c^{-1}$. The maximum densities achieved in this energy regime are between two and three times normal nuclear matter density depending on the EOS used (with a soft EOS higher densities are reached). After this compression stage the matter flows out of the central region. Therefore the central density decreases steadily to zero.

The same behaviour can be observed in figure 10 (middle) for the transverse kinetic 'temperature', which is defined as $T_{\perp}=\left(\left\langle p_{x}^{2}\right\rangle+\left\langle p_{y}^{2}\right\rangle\right) /(2 m)$.

Hard $\mathbf{n}-\mathbf{n}$ collisions lead to the degradation of the longitudinal momenta into transverse degrees of freedom, to the build-up of the compression zone and to the complete stopping of the system (figure 10 (bottom)). The average number of $\mathbf{n}-\mathbf{n}$ collisions per nucleon is plotted as a function of time for different $n-n$ cross sections. Almost all collisions take place in the time interval when the 'temperature' and the density are at their highest values. At the end of the compression stage $(t \approx 50$ $60 \mathrm{fm} c^{-1}$ ), practically all collisions have ceased. Hence, the following expansion and fragmentation stage is little affected by short-range interactions and the system evolves almost isentropically, although there are still a few collisions occurring within the formed fragments.

The large average collision numbers $N_{\text {coll }} / A \approx 5$ indicate the approach to local equilibrium. Kinetic models predict the thermalization of the incident momenta after only two or three collisions, depending on the beam energy [77-79].

In momentum space equilibration can be characterized by the transition of two initially separated Fermi spheres at $T=0$ to a thermalized matter distribution at rest in the $\mathrm{CM}$ frame.

The final rapidity spectra $\mathrm{d} N / \mathrm{d} Y$ for central and peripheral collisions are shown in figure 11 for the reaction $\mathrm{Au}$ (200 MeV/nucleon) + Au. In central collisions complete stopping results in a Gaussian-shaped rapidity distribution at rest in the CM frame. No remnants of the initial rapidity distributions centred around $y= \pm y_{\text {beam }}$ are left. Only a small fraction of the nucleons are stopped and suffer sufficient collisions to thermalize their initial momenta at large impact parameters $(b=7 \mathrm{fm})$. Two broad distributions, centred close to the initial rapidities of target and projectile, represent the spectator residues. They move on with little interaction. The rapidity spectra depend little on the EOS used.

The dependence of the $\mathrm{d} N / \mathrm{d} Y$ distributions on the mass of the system and on the bombarding energy is shown in figure 12: Complete stopping is observed only for massive systems, while lighter systems exhibit broad rapidity distributions even for very central collisions. The stopping power does not change much with bombarding energy, if the scaled rapidity distribution $\mathrm{d} N / \mathrm{d}\left(Y / Y_{P}\right)$ is considered $t$.

For the comparison with the plastic ball data [28] we applied an experimental

$\dagger$ This, in fact, remains true even for ultra-relativistic energies: The scaled baryon rapidity distributions for lighter systems ( $\mathrm{Si}+\mathrm{Si}$ at $15 \mathrm{GeV} /$ nucleon and $\mathrm{S}+\mathrm{S}$ at $200 \mathrm{GeV} /$ nucleon) do indeed show the same plateau shape as observed here (for $\mathrm{Ne}$ and $\mathrm{Ca}$ ), both theoretically [84] and experimentally [86, 87]. Also interesting is the theoretical observation that a remarkable part of the baryons is pushed to the mid-rapidity zone at energies up to $0.8 \mathrm{TeV} /$ nucleon [85]. 


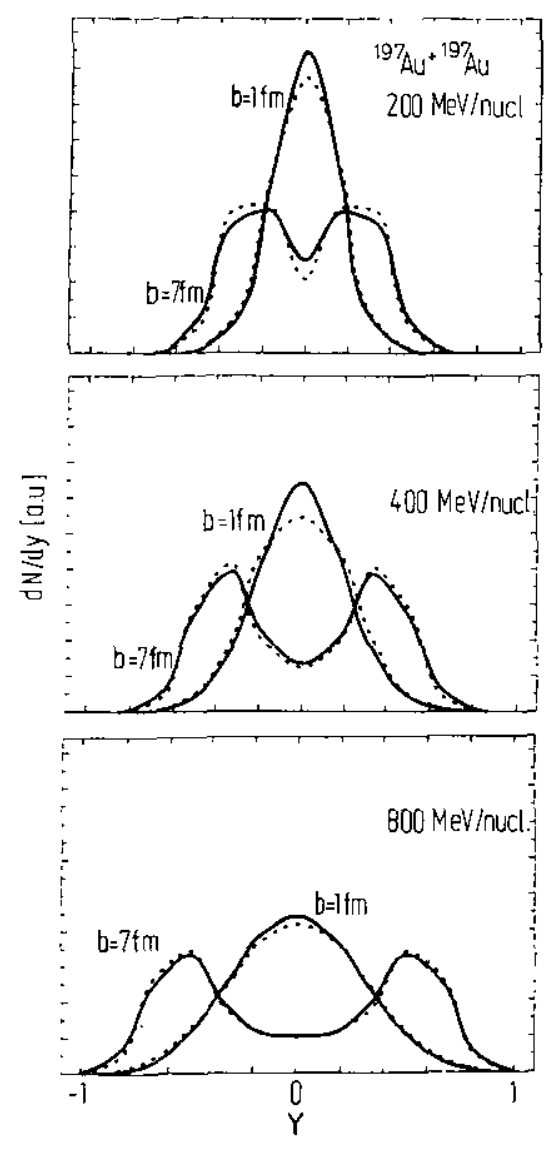

Figure 11. Rapidity distributions for the system ${ }^{197}$ Au (200 (top), 400 (middle) and 800 (boliom) $\mathrm{MeV} /$ nucleon, $b=1,7 \mathrm{fm})+{ }^{197} \mathrm{Au}$ for a hard (H) and a soft (S) Eos. Note that the rapidity distributions do not depend on the EOS.
${ }^{A} X\left(800 \mathrm{MeV} /\right.$ nucl., $\left.b=0.25 b_{\max }\right) \cdot{ }^{A X}$



$A u(b=3 \mathrm{fm})+A u$

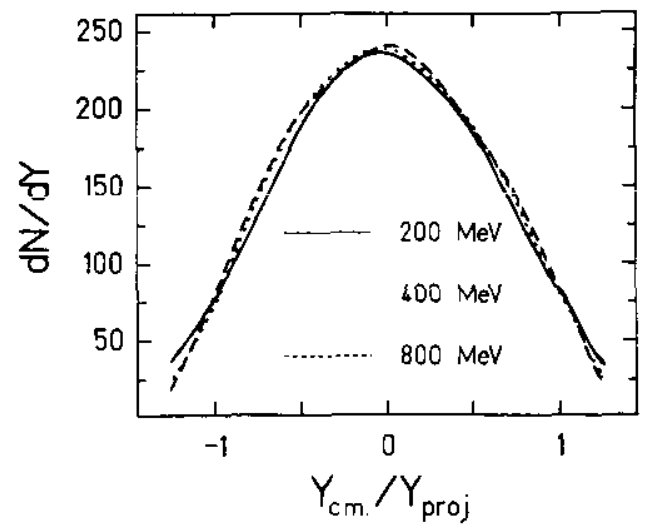

Figure 12. Mass (top) and energy (bottom) dependence of the rapidity distributions for the indicated systems. Complete stopping is observed only for massive systems at all energies investigated.

efficiency filter. Only emitted protons with an energy $E>25 \mathrm{MeV}$ and emission angles between $2.5^{\circ}$ and $160^{\circ}$ are plotted in the calculated distributions. Figure 13 shows the QMD calculations (right-hand column) compared with the plastic ball data (left-hand column) for different systems at $400 \mathrm{MeV} /$ nucleon. The protons have been determined with a spanning tree cluster algorithm [32,51, 52]. The shape of the distributions seems to be identical for all systems. However, this insensitivity is only due to the selection of protons (light clusters are predominantly seen at the projectile and target rapidities [51]). For the smaller systems there is more transparency, because the ratio of the mean free path to the diameter of the system is not small enough. The filter furthermore cuts out those particles which remain at target and projectile rapidities, because of their low energy and small angles in the 
Plastic Ball Data MUL6 $Z=1 \quad$ QMD $400 \mathrm{MeV} /$ nucl. $Z=1$

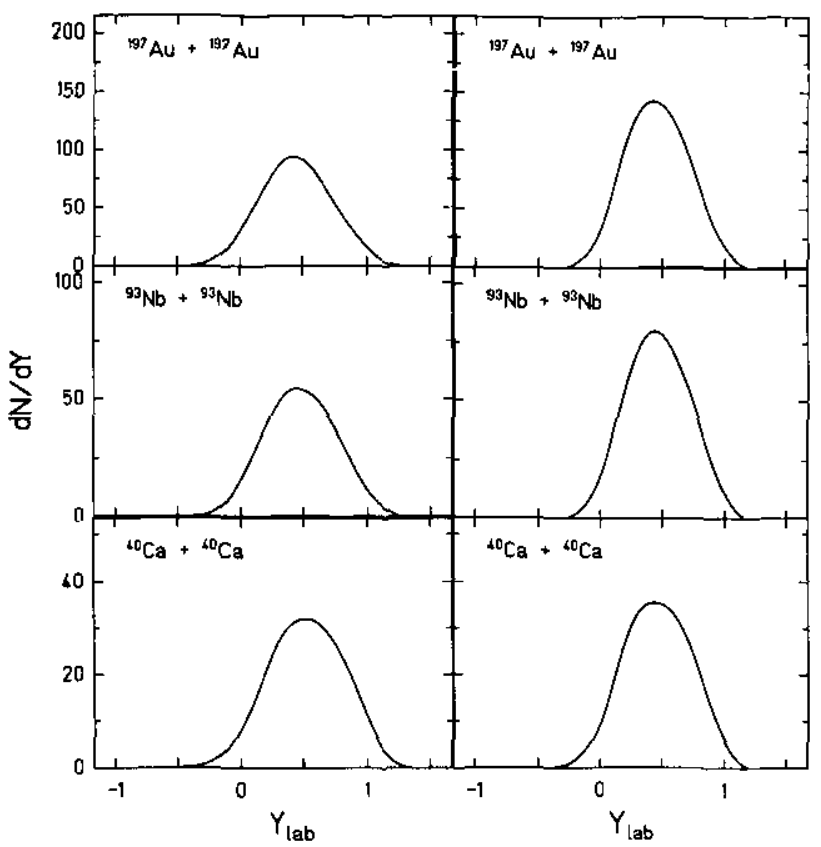

Figure 13. Mass dependence of the proton rapidity distributions. The OMD results (right) are compared with the plastic ball data (left). Due to the efficiency cuts of the plastic ball fitter all distributions (even those for the light systems) look Gaussian-shaped (see, however, figure 12).

laboratory frame respectively. Thus the plateau shapes of the unfiltered distributions only appear to be Gaussian-shaped because of the efficiency cuts. Non-negligible distortions of the mid-rapidity yields have been reported for the very heavy sytem by the plastic ball collaboration [22-29]. They are due to enhanced double-hit and cross-talk probabilities in this high track density environment. Only detector systems of even higher granularity, e.g. the GSI $4 \pi$ detector system, can avoid these problems.

The sensitivity of the nuclear stopping to the potential employed and to the effective scattering cross section has also been investigated. Figure 14 shows that the influence of the mean field on the longitudinal flow is relatively small. However, the scattcring cross section is vital for the stopping power. By using the free cross section (without Pauli blocking) the classical collision numbers double. This yields an increase in the stopping power. A global reduction of the scattering cross section with $\sigma^{\text {eff }}=0.7 \sigma^{\mathrm{UU}}$ (SIM) yields double peaked $\mathrm{d} N / \mathrm{d} Y^{\prime}$ distributions with peaks closer to the rapidities of the projectile and target. The double pcak structure originates from the remnants of the projectile and target spectators. It indicates an incomplete stopping of the incident nuclei. One should, however, keep in mind that a more complex functional dependence of $\sigma^{\text {eff }}$, e.g. on $\rho$ and $T$, could render the systematics of the interplay between the effective scattering cross section and the longitudinal and transverse flow much more complicated. The $\mathrm{d} N / \mathrm{d} Y$ distribution of the soft EOS with the Uehling-Uhlenbeck cross section $\sigma^{\mathrm{UU}}(\mathrm{S})$ lies between the curves $\mathrm{S}$ and SIM. The MDI (SM) modifies the stopping power slightly. The evident differences between 


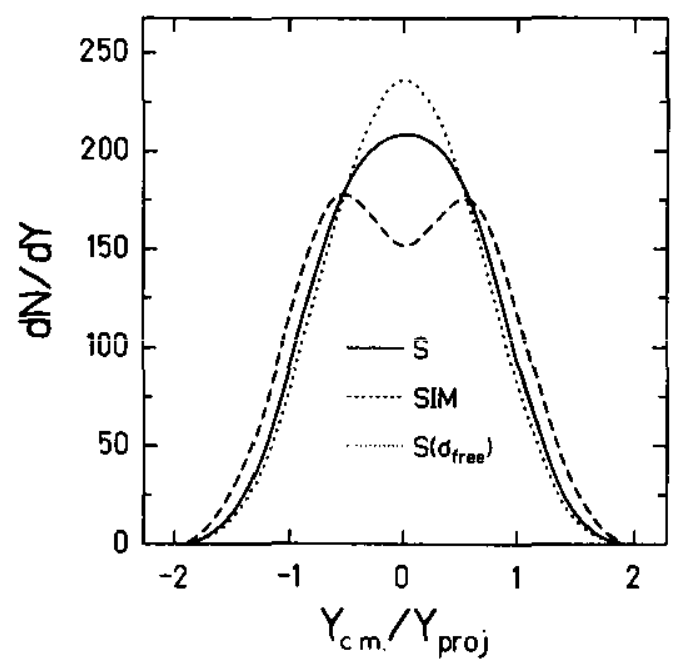

Figure 14. The influence of the different interactions on the rapidity distributions $\mathrm{d} N / \mathrm{d} Y$ (all particles included) obtained from the reaction ${ }^{197} \mathrm{Au}$ (200 MeV/nucleon, $b=3 \mathrm{fm})+{ }^{197} \mathrm{Au}$. The $\mathrm{d} N / \mathrm{d} Y$ distributions depend strongly on the $n-n$ cross sections used!

the $\mathrm{d} N / \mathrm{d} Y$ spectra obtained from the cases $\sigma^{\text {eff }}$ and $\sigma^{\mathrm{UU}}$ can be exploited for the experimental determination of $\sigma^{\text {eff }}$ from the $\mathrm{d} N / \mathrm{d} Y^{\prime}$ spectra for different systems at different energies (see, e.g., $[51,66]$ ).

The measured rapidity spectra for the systems $\mathrm{Ar}(1200 \mathrm{MeV} / \mathrm{nucleon})+\mathrm{BaI}_{2}$ and $\mathrm{KCl}$ are in good agreement with vuU calculations [67], which use the free scattering cross section $\sigma^{\mathrm{UU}}$. A reduced-or increased- $\sigma^{\text {eff }}$ fails to reproduce these data, just as the $\mathrm{Au}+\mathrm{Au}$ data show no dip.

\subsection{Transverse momentum transfer and the concept of a temperature'}

The interplay between the longitudinal and the transverse flow can be seen clearly by inspection of the double differential cross section $\mathrm{d}^{2} N / \mathrm{d} y \mathrm{~d} p_{\perp}$ in figure 15 . It is shown for different impact parameters for the reaction Au (650 Mív/nucieon) + Au.

The final nucleon distribution is centred around the original beam and target rapidities and momenta for peripheral collisions (at $b=7 \mathrm{fm}$ ). With decreasing impact parameter the two peaks come closer to each other, merge at $b=3 \mathrm{fm}$ and form a single 'equilibrated' source for central collisions $(b=1 \mathrm{fm})$. The stopping of the incoming matter is closely related to the transverse momentum transfer. The transverse momenta increase with decreasing impact parameter, indicating the thermalization of the initial beam momenta (The 'thermalization' is studied quantitively in the next section.)

The absolute width of the final momentum distribution is shown in figure 16, where we compare the longitudinal and transverse 'temperatures' of the global system, defined by $T_{\perp}=\left(\left\langle p_{x}^{2}\right\rangle+\left\langle p_{y}^{2}\right\rangle\right) / 2 m$ and $T_{\|}=\left\langle p_{z}^{2}\right\rangle / m$.

Figure 16 (top and middle) shows the change of the relative magnitude of the longitudinal and the transverse temperature when the impact parameter changes from 1 to $3 \mathrm{fm} c^{-1}$. Here a hard EOS is used, for the soft EOS the two components of the temperatures are identical. This demonstrates a substantial sensitivity of the directed sidewards flow to the potential (EOS). The transverse flow is less pronounced for the 
soft EOS, in agreement with the results obtained with the vUU approach [30, 33]. However, this result does not give sufficient information to conclude that the system is equilibrated, as we will discuss in the next section.

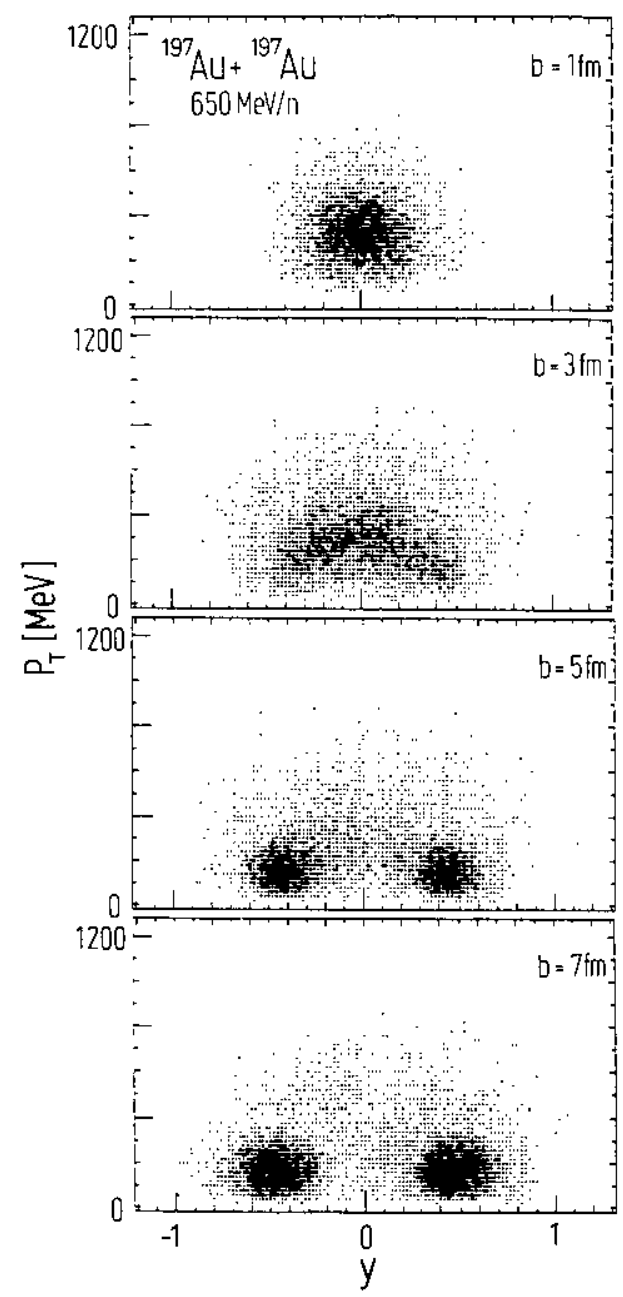

Figure 15. Invariant double differential momentum distribution $\mathrm{d}^{2} \mathrm{~N} / \mathrm{d} y \mathrm{~d} p_{\perp}$ for the reaction ${ }^{197} \mathrm{Au}$ (800 MeV/nucleon, hard EOS) $+{ }^{197} \mathrm{Au}$ at different impact parameters as indicated.

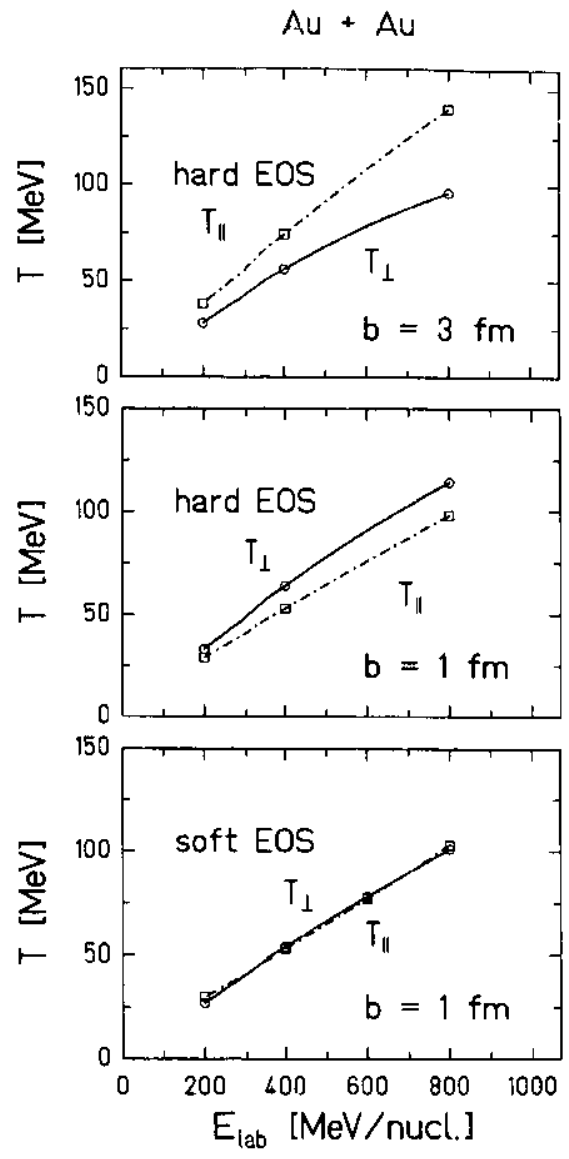

Figure 16. Excitation function of the apparent 'temperatures' in the system ${ }^{197} \mathrm{Au}(b=1$ and $3 \mathrm{fm})+{ }^{197} \mathrm{Au}$. The mean quadratic momenta in beam direction $T_{\|}$(broken curves) and in transverse direction $T_{\perp}$ (full curves) are displayed for a hard (top and middle) and a soft (bottom) Eos, respectively in almost central collisions. These 'temperatures' are determined via the corresponding mean quadratic momenta in the $\mathrm{CM}$ frame: $T_{y}=(1 / m)\left\langle p_{z}^{2}\right\rangle, T_{\perp}=(1 / 2 m)\left[\left\langle p_{x}^{2}\right\rangle+\left\langle p_{y}^{2}\right\rangle\right]$. Note the substantial sensitivity of the directed sidewards flow on the Eos and the impact parameter. 


\section{The importance of non-equilibrium effects and the transport coefficients}

\subsection{The pressure and equilibration process}

Let us now investigate to what extent thermodynamic concepts, in particular local equilibrium, are justified. The local equilibrium concept forms the basis of ideal fluid dynamics. The assumption of global equilibrium forms the basis of most statistical models.

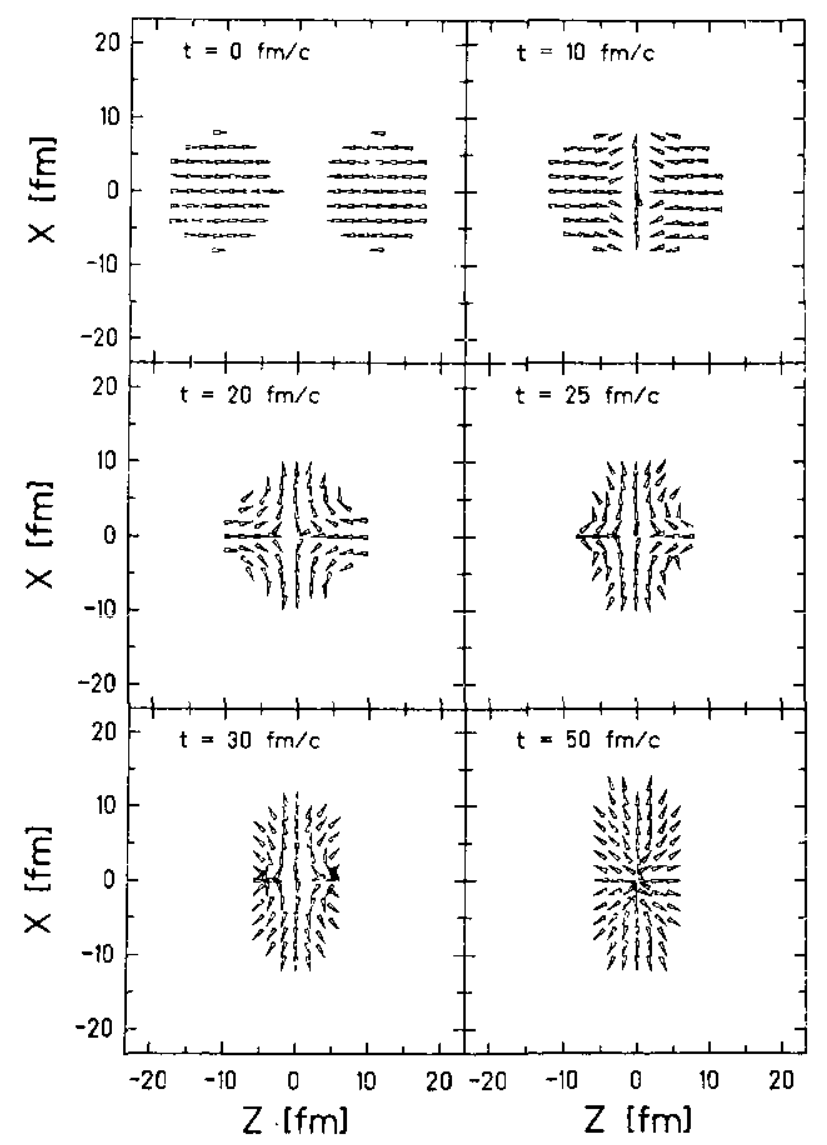

Figure 17. Time evolution of the reaction ${ }^{197} \mathrm{Au}(200 \mathrm{McV} / \mathrm{nucleon}, b=0 \mathrm{fm})+$ ${ }^{197} \mathrm{Au}$ in velocity space. Observe the formation of a shock fronl where the nucleons are deflected to sidewards angles.

First we investigate the evolution of the reaction $\mathrm{Au}(200 \mathrm{MeV} /$ nucleon, $b=0 \mathrm{fm}$ ) + Au in velocity space. Figure 17 shows the highly anisotropic initial configuration. When the nuclei start to touch each other in coordinate space the nucleons in the overlap region are deflected to sidewards angles. This is a beautiful microscopic proof of the behaviour predicted by hydrodynamics $[1-18]$, i.e. a zone of shocked matter is formed.

The time evolution of the density along the beam direction (figure 18) reveals this scenario. First a very small high density region is formed in the centre of the reaction. This region then expands due to the further flux of matter from projectile 

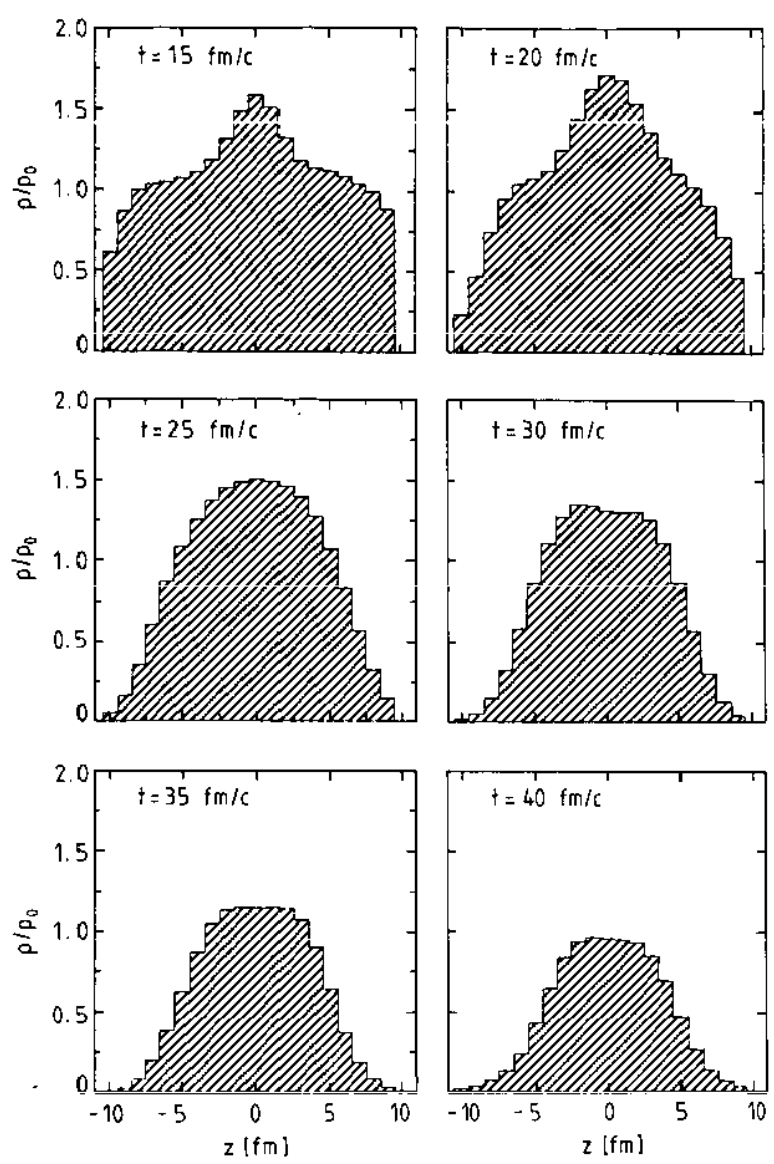

Figure 18. Density profiles along the beam direction for the same reaction as in figure 17 . A plateau with approximately 1.5 times normal nuclear matter density is formed.

and target. A plateau with density about 1.5 times normal nuclear matter density is formed. However, we have to clarify whether this zone is equilibrated or not. The local degree of isotropy $R$ is defined as $R=\left\langle p_{\mathrm{T}}^{2}\right\rangle / 2\left\langle p_{\mathrm{L}}^{2}\right\rangle$, where $p_{\mathrm{T}}$ and $p_{\mathrm{L}}$ are taken in the rest frame of the matter element under consideration. Then $R \approx 0$ means total anisotropy which is characteristic for the first stage of a collision in which the two nuclei just touch and no transverse momentum has yet been transferred. An isotropic momentum distribution would lead to $R=1$. Note, however, that $R=1$ is a necessary, but not necessarily sufficient condition for equilibration.

For heavy systems one expects to be close to a local equilibrium situation, where viscous hydrodynamics is applicable. The values of $R(x, z)$ in the reaction plane $(x, z)$ are shown in figure 19 . The two nuclei are totally thermalized $(R=1)$ initially in their respective rest frames. Early in the reaction the interpenetrating nuclei yield a strong anisotropy in this overlap region. The remaining parts of the projectile and target remain equilibrated. At the time of full overlap the central density and temperatures have reached their maximal values. Here $R \approx 0.5$ still appears to be small. When the matter starts to decompress near equilibration is observed in this central zone. 
$200 \mathrm{MeV} /$ nucl.
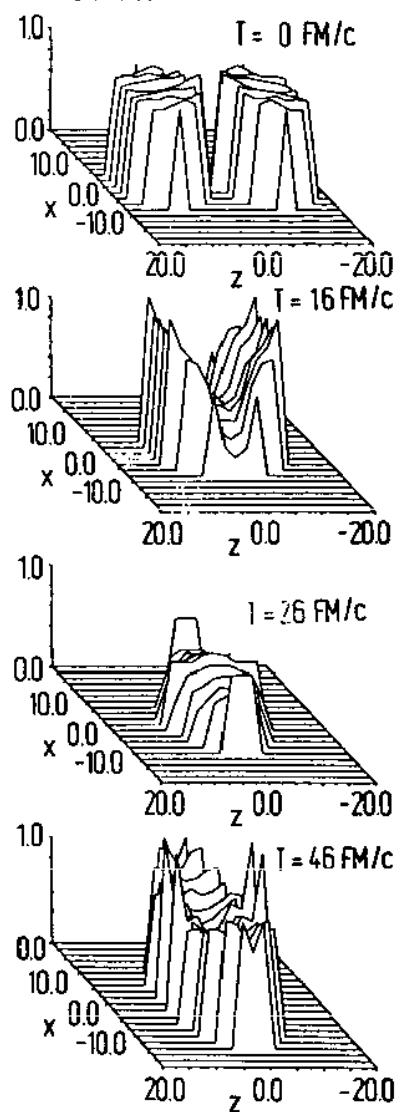

$800 \mathrm{MeV} / \mathrm{nudl}$.
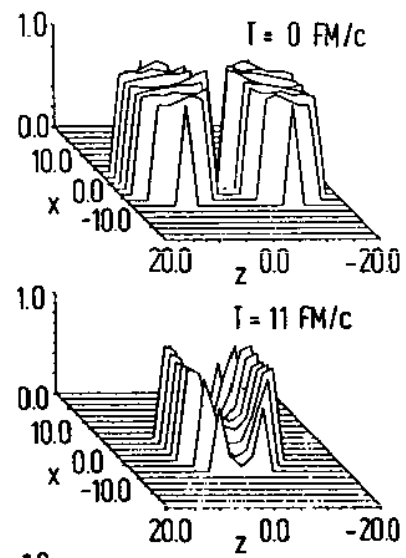

$\Gamma=19 \mathrm{FM} / \mathrm{C}$


Figure 19. Snapshots of the time evolution of the thermalization ratio $R=$ $\left\langle P_{\perp}^{2}\right\rangle /\left(2\left\langle P_{\|}^{2}\right\rangle\right)$ over the reaction plane in very central collisions. A density cut has been introduced in order to avoid strong statistical fluctuations $\left(\rho>0.1 \rho_{0}\right)$. Equilibra. tion is observed at the end of the reaction in the central zone.

Figure 20 shows the time evolution of $R$ at the origin $(x=y=z=0)$ for the reaction $\mathrm{Au}(b=0 \mathrm{fm})+\mathrm{Au}$ at 200 and $800 \mathrm{MeV} /$ nucleon bombarding energy. The display is for three different cases, showing $R$ for all nucleons (full curve), for those nucleons which have suffered at least one (broken curve) or even two (dotted curve) collisions. Local equilibrium is clearly not achieved for the total system, while the participant component equilibrates better $(R \approx 0.8-0.9$ ).

\subsection{The evaluation of the stress tensor: pressure and viscosity}

Information about the thermalization process can also be obtained from the stress tensor

$$
P_{k l}(\boldsymbol{r}, t)=\int \mathrm{d}^{3} p f(\boldsymbol{p}, \boldsymbol{r}, t)\left(p_{k}-\left\langle p_{k}(\boldsymbol{r}, t)\right\rangle\right)\left(v_{l}-\left\langle v_{l}(\boldsymbol{r}, t)\right\rangle\right) \quad k, l=1,3 .
$$

The interaction part of $P_{i k}$ has been excluded from the analysis, because $P_{i k}$ is to be compared with the Newtonian ansatz used in viscous fluid dynamics $[6,8,10$, 


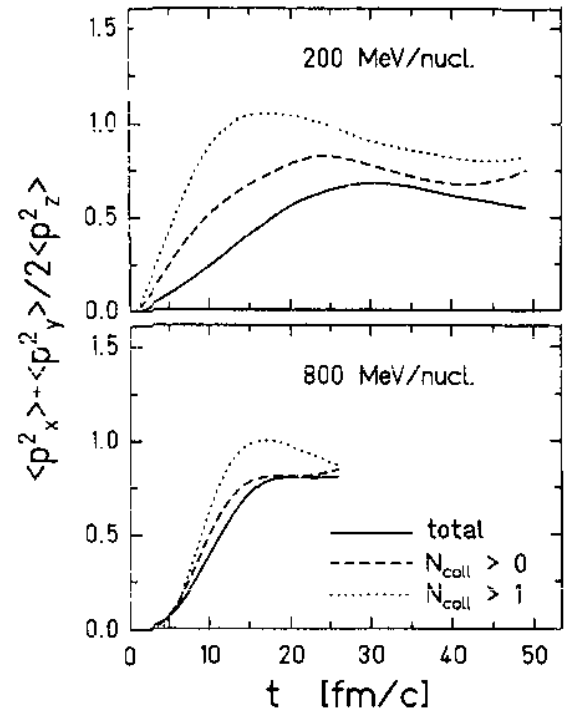

Figure 20. Ratio of the mean quadratic momenta at the origin of the $\mathrm{cm}$ frame in the transverse and longitudinal directions for central $\mathrm{Au}+\mathrm{Au}$ collisions at $200 \mathrm{MeV} / \mathrm{nucleon}$ (top) and $800 \mathrm{MeV} / \mathrm{nucleon}$ (bottom) for all particles (full curves), for particles which have collide at least once (broken curves) and more than once (dotted curves). The participant component equilibrates much better than the spectators. This behaviour is more pronounced at lower energies.

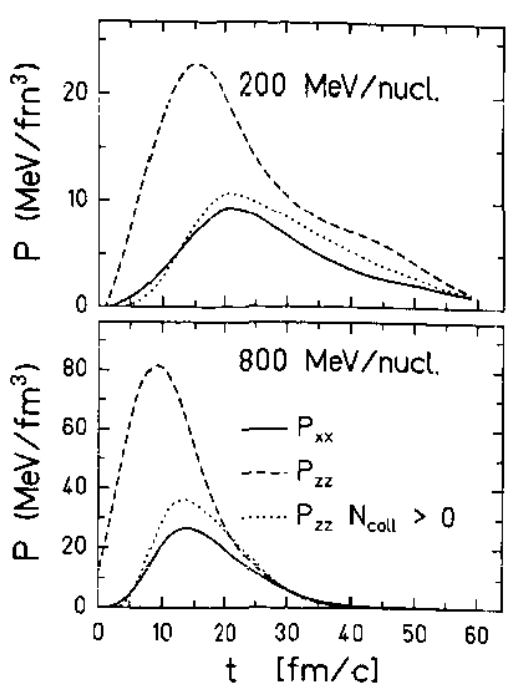

Figure 21. 'lime dependence of the kinetic pressure in the centre of the reaction for the system Au (200 (top), 800 (bottom) MeV/nucleon, $b=0 \mathrm{fm}$ ) + Au. The broken curves correspond to the $x x$ and $y y$ components of the stress tensor, full curves to the $z z$ component. A thermal (chain curves) and a non-thermal part (dotted curves) are defined for nucleons which have suffered at least one collision.

76]. For the local interactions used here $P_{i k}$ is trivially isotropic. That means the sum of the kinetic and interaction pressure will always appear to be more isotropic than the kinetic pressure (stress tensor) alone. From the QMD distribution function (see equation (2)) one obtains

$$
\begin{aligned}
P_{k l}(\boldsymbol{r}, t)= & \frac{1}{(2 \pi L)^{3 / 2}} \sum_{i=1}^{N} \exp \left\{-\frac{\left(\boldsymbol{r}-\boldsymbol{r}_{i 0}(t)\right)^{2}}{2 L}\right\} \\
& \times\left(p_{i 0}-\langle p(r, t)\rangle\right)_{k}\left(\left(p_{i 0} / m_{i}\right)-\langle v(r, t)\rangle\right)_{l} .
\end{aligned}
$$

Figure 21 shows the time evolution of the different components of the stress tensor. The maxima of both the $z z$ component (full curve) and the $x x$ component (broken curve) appear at the same time as the maxima of the temperatures and the central densities. However, the absolute values of these components differ from each other, they approach one common value towards the end of the reaction. This again reflects the importance of treating the non-equilibrium aspects during the early course of the reaction.

On the other hand, for fireball nucleons the $z z$ component of the stress tensor (dotted curve) is almost identical to the $x x$ component, i.e. the fireball is near thermal equilibrium.

Hence, the basic assumption of the three-fluid model $[9,15]$ is justified: each 


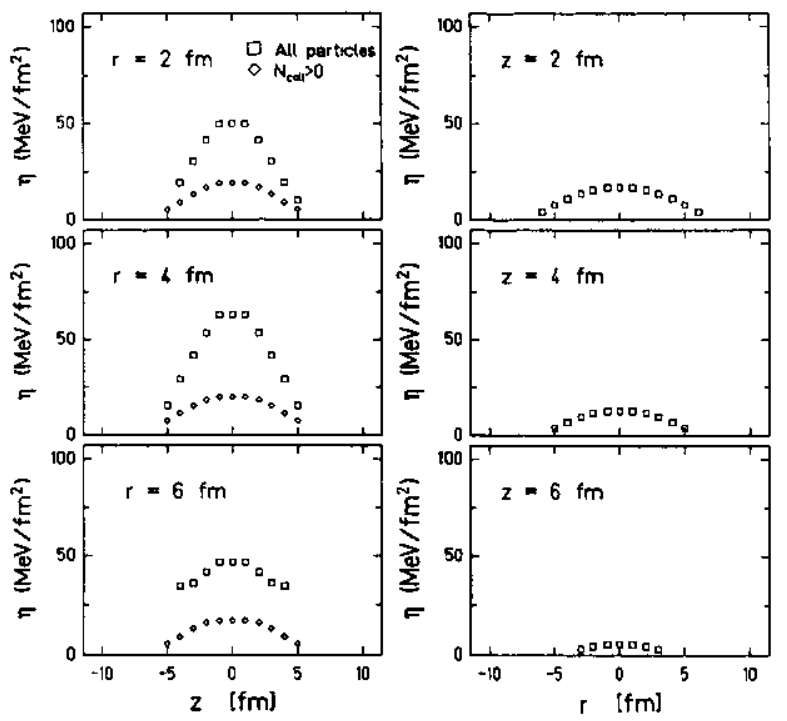

Figure 22. Shear viscosity coefficient $\eta$ extracted from QMD calculations in the reaction Au (200 MeV/nucleon, $b=0 \mathrm{im})+A u$ al $t=20 \mathrm{fm} c^{-1}$ along the beam direction $z$ (left) and in (ransverse direction $r$ (right). Note the apparent difference between the viscosity coeflicent in the longitudinal and transverse directions.



Figure 23. Same as figure 22 tor $800 \mathrm{MeV} / \mathrm{nuclcon}$ (evaluated at $10 \mathrm{fm} \mathrm{c}^{-1}$ ).

of the three components closely approach thermal equilibrium. The non-equilibrium effects are most important at the beginning of the reaction.

\subsection{The applicability of macroscopic models}

Can the concept of viscous fluid dynamics $[6,8,10,76]$ be applied to heavy ion 
collisions? The viscosity coeflicients $\eta$ (shear viscosity) and $\xi$ (bulk viscosity) are defined by the following Newtonian form of the stress tensor.

$$
P_{i j}=p \cdot \delta_{i j}-\eta \cdot\left(\frac{\partial V_{i}}{\partial x_{j}}+\frac{\partial V_{j}}{\partial x_{i}}-\delta_{i j} \frac{2}{3} \cdot \partial V\right)-\delta_{i j} \cdot \zeta \cdot \partial V .
$$

The viscosity and its density and temperature dependence serve as a constitutive equation for hydrodynamical calculations. In a microscopic model these viscosity cocfficicnts can bc dctcrmincd by comparing the exact pressure tensor (equation (9)) to the Newtonian form (equation (11)) [80]. Furthermore this Newtonian ansatz itself can be checked. This means that unique coefficients $\eta$ and $\xi$ should be found so that all, in general anisotropic, components of the stress tensor obey relation (11) with the same coefficients. However, we find here that the coefficients for the longitudinal components of the stress tensor are about a factor of three larger than the coeflicient for the transverse component.

Figure 21 shows the shear viscosity coefficient as a function of both the transverse (denoted by $r$ ), and the longitudinal (denoted with $z$ ) distance from the origin, extracted for the reaction $\mathrm{Au}(200 \mathrm{MeV} /$ nucleon, $b=0 \mathrm{fm})+\mathrm{Au}$, at $20 \mathrm{fm} c^{-1}$ (note the cylindrical symmetry of the system). The left-hand column shows the longitudinal viscosity coefficient $\eta_{r z}$ for all particles and for particles which have collided at least once. $\eta_{r z}$ reaches maximum values around $60 \mathrm{MeV} \mathrm{fm}^{-2} c^{-1}$ in the highest density region, if all particles are considered. It drops to zero at larger distances from the centre due to the density and temperature dependence. In contrast, the participant component reaches values of approximately $20 \mathrm{MeV} \mathrm{fm}^{-2} c^{-1}$ only. The right-hand column shows the transverse component $\eta_{x y}$, which reaches maximum values of $20 \mathrm{MeV} \mathrm{fm}{ }^{-2} c^{-1}$, which are nearly identical to the 'participant' component. The same situation is found for higher energies (sce figure 23). A maximum viscosity coefficient of $130 \mathrm{MeV} \mathrm{fm}^{-2} c^{-1}$ is needed in order to get a proportionality between the stress tensor and velocity gradient at $800 \mathrm{MeV} /$ nucleon. For the transverse component a maximum value of $20 \mathrm{MeV} \mathrm{fm}^{-2} c^{-1}$ is found. A higher value of the viscosity coefficient is found in the longitudinal direction.

Previous one- [6], two- [8] and three-dimensional [10] viscous hydrodynamical calculations used much smaller viscosity coefficients than those obtained by kinetic theories. These low values were motivated by fission fragment spectra, calculated by Hofmann and Nix [81]. However, recently hot fission experiments have lead to much higher extracted viscosity coefficients [82] in accordance with the estimates of kinetic theories [72, 73].

Recent hydrodynamical calculations [76] show that only such high viscosity coefficients can explain the flow and bounce-off data. However, viscosity coefficients of $60 \mathrm{MeV} \mathrm{fm}^{-2} c^{-1}$ yield better agreement with the experimentally observed in-plane sidewards, while the off-plane squeeze-out are to be reproduced with $\eta=40 \mathrm{MeV} \mathrm{fm}{ }^{-2} c^{-1}$ (see also [63]). This is in qualitative agreement with the previous results.

\section{Summary and conclusions}

We have investigated the 'stopping power' of nuclei and the thermalization process employing the OMD approach. 
The mean free path of the nucleons, $\lambda$, which has been calculated microscopically, is found to be mass independent, e.g. it can be considered as a 'material' constant. $\lambda$, however, is strongly energy dependent; it decreases with the energy, in contrast to the naive expectation based on classical kinetic theory [75], which yields an increasing $\lambda$ because $\sigma$ decreases with energy. This decrease is a genuine quantum statistical effect. In the QMD (and the VUU) model it originates from the 'Pauli blocking' of the collisions, which becomes more important at low energies, due to the occupied phase space. The 'soft' col'isions at very low $\sqrt{s}$ are found to be most strongly blocked. Even at high energies ( $1 \mathrm{GeV} /$ nucleon) more than $20 \%$ of the collisions are still blocked. Classical molecular dynamics [40-49], which do not include this quantum effect, therefore overestimate stopping, thermalization and collective flow.

The rapidity distributions only show stopping for heavy systems, independent of the energy. However if one takes only free protons and in addition the efficiency filter of the plastic ball into account, the shapes of the $\mathrm{d} N / \mathrm{d} Y$ distributions are also found to be mass independent. The rapidity distributions are very sensitive to the $n-n$ cross sections, but do not depend on the EOS.

In order to analyse the thermalization achieved in heavy ion collisions, a local analysis of macroscopic quantities, such as density, mean moments of the momenta and the pressure, have been performed. We have found that non-equilibrium aspects play an important role in heavy ion reactions. At the time when all the observables from which one wants to extract the equation of state are established, the reaction cannot be cast in a one- or two-fluid dynamical picture. A rapid approach to local equilibration is observed in a three-fluid decomposition. These components can be experimentally distinguished by their clear separation in phase space ( $Y$ and $\left.p_{\mathrm{T}}\right)$. The dynamical evolution of the reaction is dominated by the interaction of the projectile with the participants, and, scparately, by the target with the participants. The direct interpenetration of projectile and target is not observed.

The viscous three-component fluid dynamical model and anisotropic hydrodynamics [88] seem to be the only macroscopic models which can incorporate the non-equilibrium effects in a proper way.

This also points to the necessity of looking for signatures of the equation of state in those observables which are dominated by nucleons from the participant component only, because here the distortion of the observables due to 'spectators' and due to momentum-dependent effects is minimized.

\section{References}

[1] Scheid W, Müller $H$ and W Greiner 1974 Phys. Rev. Lett. 32741

[2] Baumgardı H G, Scholl J V, Sakamoto Y, Schopper E, Stöcker H, Hofmann J, Scheid W and Greiner W 1975 Z. Phys. A 273359

[3] Amsden A A, Harlow F H and Nix J R 1977 Phys. Rev. C 152059

Amsden A A, Ginocchio J N, Harlow F H, Nix J R, Danos M, Halbert E C and Smith R K 1977 Phys. Rev. Lett. 381055

[4] Amsden A A, Goldhaber A S, Harlow F H and Nix J R 1978 Phys. Rev. C 172080

[5] Stöcker H, Maruhn J A and Greiner W 1980 Phys. Rev. Lett. 44 725; 1979 Z. Phys. A 290 297

[6] Csemai L P, Lukacs B and Zimanyi J 1980 Nuovo Cimento Lett. 27111

Csemai L P and Barz H W 1981 Z Phys. A 296173

[7] Stöcker H, Csernai L P, Graebner G, Buchwald G, Kruse H, Cusson R Y, Maruhn J A and Greiner W 1981 Phys. Rev. Lett. 47 1807; 1982 Phys. Rev. C 251873

[8] Buchwald G, Csernai L P, Maruhn J A, Greiner W and Stöcker H 1981 Phys. Rev. 24135 
[9] Csemai L P, Lovas I, Maruhn J A, Rosenhauer A, Zimanyi J and Greiner W 1982 Phys. Rev. C 26 149

[10] Buchwald G, Graebner G. Theis J, Maruhn J A, Greiner W and Stöcker H 1983 Phys. Rev. C 28 11!0

[11] Russkikh 1983 Son J. Nucl. Phys. 38 381; 1986 Sov. J. Nucl. Phys. 44961

[12] Buchwald G, Gräbner G, Theis J, Maruhn , W. Greiner W and Stöcker H 1984 Phys. Rev. Lett. 52 1594

[13] Ivanov Y B and Satarov L M 1985 Nucl. Phys. A 433713

[14] Clare R B and Strottman D 1986 Phys. Rep. 141172

[15] Rosenhauer A, Maruhn J A, Greiner W and Csernai L P 1987 Z. Phys. A 326213

[16] Mishustin I N 1989 Nucl Phys. A 494595

[17] Satarov L M 1990 Sov. J. Nucl. Phys. 52264

[18] Stöcker H and Greiner W 1986 Phys. Rep. 137

[19] Gustafsson H A et al 1984 Phys. Rev. Lett. 521590

[20] Renfordt R E 1984 Phys. Rev. Lett. 53763

[21] Beauvis D 1983 Phys. Rev. C 272443

[22] Ritter H G et al 1985 NucL Phys. A 447 3c

[23] Doss K G B et al 1986 Phys: Rev: Lett. 57302

[24] Doss K G B et al 1987 Phys. Rev: Lett. 592720

[25] Jacak B V et al 1987 Phys. Rev. C 351751

[26] Kampert K H 1989 J. Phys. G: NucL Part. Phys. 15 691-740

[27] Gutbrod H H, Poskanzer A M and Ritter H G 1989 Rep. Prog. Phys. 521267

[28] Gutbrod H H, Kampert K H, Kolb B W. Poskanzer A M, Ritter H G and Schmidt H R 1990 Z. Phys. A $33757-69$

[29] Schmidt H R GS/ Report 91-03

[30] Kruse H, Jacak B V and Stöcker H 1985 Phys. Rev: Lett. 54289

[31] Molitoris J J and Siöcker H 1985 Phys. Rev: C 32 346; 1985 Phys. Lett. 162B 47

[32] Kruse H, Jacak B V, Molitoris J J, Westiall G D and Stöcker H 1985 Phys. Rev. C 311770

[33] Molitoris J J, Stöcker H and Winer B L 1987 Phys. Rev. C 36220

[34] Gregoire C, Remaud B, Sebille F and Vinet L, 1987 Nucl. Phys. A 465317

[35] Bertsch G F, Kruse H and Das Gupta S 1984 Phys. Rev. C 29673

[36] Aichelin J and Stöcker H 1985 Phys. Lett. 16359

[37] Aichelin J and Bertsch G F 1985 Phys. Rev: C 311730

[38] Gale C, Bertsch G F and Das Gupta S 1987 Phys. Rev. C 351666

[39] Bertsch G F, Lynch W G and Tsang M B 1987 Phys. Lett. 18913 384

[40] Bodmer A and Panos C N 1977 Phys. Rev: C 151342

[41] Wilets L, Henley E M, Kraft M and Mackellar A D 1987 Nucl. Phys. A 282341

[42] Wilets L, Yariv Y and Chestnut R 1978 Nucl. Phys. A 301359

[43] Callaway D J E, Wilets L and Yariv Y 1979 Nucl. Phys. A 327250

[44] Bodmer A R, Panos C, MacKellar A D 1980 Phys: Rev: C 221025

[45] Molitoris J J, Hoffer J B, Kruse and Stöcker H 1984 Phys. Rev. Lett. 53899

[46] Kiselev S M and Pokroskil Yu E 1983 Sov. J. Nicl. Phys. 3846

[47] Kiselev S M 1984 Sol: J. Nuch Phys. 3918

[48] Kiselev S M 1986 Sov. J. NucL Phys. 44610

[49] Schlagel T J and Pandharipande V R 1987 Phys. Rev: C 36192

[50] Aichelin J and Stöcker $\mathrm{H}$ ly86 Pitys. Lett. 176 A 14

[51] Peilert G, Rosenhauer A, Aichelin J, Stöcker H and Greiner W 1988 Mod. Phys. Lett. A 3 459; 1989 Phys. Rev. C 391402

[52] Aichelin J, Peilert G, Bohnet A, Rosenhauer A, Stöcker H and Greiner W 1988 Phys. Rev. C 37 2451

[53] Aichelin J 1991 Phys. Rep. 202233

[54] Berenguer M, Hartnack C. Peilert G, Rosenhauer A, Schmidı W, Aichelin J, Marulnn J A, Greiner W and Stöcker H 1989 Proc. Winter School on Nuclear Physics (Les /Houches, France)

[55] Hartnack C 1989 Diploma Thesis Universitäı, Frankfurt unpublished

[56] Beauvais G E, Boal D H and Wong J C K 1987 Phys: Rev. C 36192

[57] Boal D H and Glosli J M 1988 Phys. Rev. C 38 1870; 1987 Phys. Rev: C 382621

[58] Boal D H, Gosli J H and Wicentowich C 1989 Phys. Rev. C 40601

[59] Botermans W and Malfiet R 1986 Phys. Lett. $171 \mathrm{~B} 22$ 
[60] ter Haar B, Malfliet R and Botermans W 1986 Phys. Lett. 172B 10; 1987 Phys. Rep. 149207

[61] Cugnon J, Lejeune A and Grange P 1987 Phys. Rev. C 35861

[62] Ohtsuka N, Linden R, Faessler A and Malik F B 1987 Nucl Phys. A 465550

[63] Ainsworth T L, Baron E, Brown G E, Cooperstein J and Prakash M 1987 Nucl. Phys. A 464740

[64] Schürmann B 1988 Mod. Phys. Lett. A 121137

[65] Schürmann B and Zwermann W 1987 Phys. Rev. Lett. 592848

[66] Cusson R Y, Reinhardt P G, Molitoris J J, Stöcker H, Strayer M, Greiner W 1985 Phys. Rev. Lett. 552786

[67] Keane D, Chu S Y, Fung S Y, Liu Y N, Qiao L J, VanDalen G, Vient M, Wang S, Molitoris J J and Stöcker H 1987 Proc. VIllth High Energy Heavy lon Study (LBL Berkeley); 1988 Phys. Rev. C $37 \quad 1447$

[68] Ayik S and Gregoire C 1988 Phys. Lett. 212B 269; 1990 Nucl. Phys. A 513187

[69] Randrup J and Remaud B 1990 Nucl. Phys. A 514339

[70] Feldmeier H 1990 Nucl. Phys. A 515147

[71] Dorso C, Duarte S and Randrup J 1987 Phys. Lett. 188B 287

[72] Peilert G, Randrup J, Stöcker H and Greiner W 1991 Phys. Lett. B 260271

[73] Bodmer A R 1978 Proc. Symp. on Relativistic Heavy-lon Research (GSI Darmstadt); Preprint Argonne National Laboratory

[74] Danielewicz P 1984 Phys. Lett. 1461 168

[75] Sobel M I, Siemens P J, Bondorf J B and Bethe H A 1975 Nucl. Phys. A 251502

[76] Schmidt W 1989 Thesis Universität Franklurt; 1989 GS/ Report 89.16; 1989 Proc. NATO Advanced Study Institute on the Nuclear Equation of State (Peniscola, Spain, 1989): Part A: Discovery of Nuclear Shockwaves and the EOS (NATO ASI Series, Series B: Physics 216A) ed W Greiner and H Stöcker p 197

[77] Uehling E A and Uhlenbeck G E 1933 Phys, Rev. 43 552; 1979 Phys. Rev. 46917

[78] Randrup J 1979 Nucl. Phys. A 314429

[79] Cugnon J, Mitzutani T and Vermeulen J 1981 NucL Phys. A 352505

[oo] Roseñhauer A, Csernai L $P$, Mánihü J A, Greiner W 1904 Rhys. Sci: 3045

[81] Cugnon J and Hote D L 1984 Proc. 7th High Energy Heavy Ion Study, GSI Darmstadt (GSI Report 85) р 253

[82] Hofmann H and Nix J R 1983 Phys. Leth. 122B 117

[83] Paul P private communication; Preprim State University of Stony Brook

[84] Sorge H, Stöcker H and Greiner W 1989 Ann. Phys., NY 192266

[85] von Keitz A, Winckelmann 1, Jahns A, Sorge H, Stöcker H and Greiner W 1991 Preprint University of Frankfurt, UFTP 255; 1992 Phys. Letl. B submilted

[86] Bloomer M 1990 Thesis MIT

[87] Wenig S 1990 Thesis Universität of Frankfurt

[88] Neise L W 1990 Thesis University of Frankfurt; GS/ Report 90-24 